This is a self-archived version of an original article. This version may differ from the original in pagination and typographic details.

Author(s): Ahokas, Essi; Ihalainen, Johanna; Kyröläinen, Heikki; Mero, Antti

Title: Effects of Water Immersion Methods on Postexercise Recovery of Physical and Mental Performance

Year: 2019

Version: Accepted version (Final draft)

Copyright: (c) 2019 by the National Strength

Rights: In Copyright

Rights url: http://rightsstatements.org/page//nC/1.0/?language=en

Please cite the original version:

Ahokas, E., Ihalainen, J., Kyröläinen, H., \& Mero, A. (2019). Effects of Water Immersion Methods on Postexercise Recovery of Physical and Mental Performance. Journal of Strength and Conditioning Research, 33(6), 1488-1495. https://doi.org/10.1519/JSC.0000000000003134 


\section{Effects of water immersion methods on post-exercise recovery of physical and mental performance}

Essi K. Ahokas,1 Johanna K. Ihalainen,1,2 Heikki Kyröläinen,1 and Antti A. Mero1

1Unit of Biology of Physical Activity, University of Jyväskylä, Jyväskylä, Finland; and 2Department of Health Sciences, Swedish Winter Sports Research Center, Mid Sweden University, Östersund, Sweden 


\begin{abstract}
The aim of this study was to compare the effectiveness of three water immersion interventions performed after active recovery compared to active recovery only on physical and mental performance measures and physiological responses. The subjects were physically active men (age 20-35 years, mean \pm SD $26 \pm 3.7$ years). All participants performed a short-term exercise protocol, including maximal jumps and sprinting. Four different recovery methods (10 minutes) were used in random order: cold water immersion (CWI, 10 ${ }^{\circ} \mathrm{C}$ ), thermoneutral water immersion $\left(\mathrm{TWI}, 24^{\circ} \mathrm{C}\right.$ ), contrast water therapy $\left(\mathrm{CWT}\right.$, alternately $10^{\circ} \mathrm{C}$ and 38 ${ }^{\circ} \mathrm{C}$ ). All of these methods were performed after an active recovery (10 minutes bicycle ergometer; heart rate $120-140 \mathrm{bpm}, 60-73 \%$ from age-calculated maximum heart rate), and the fourth method was active recovery (ACT) only. Within 96 hours after exercise bouts, recovery was assessed via a $30 \mathrm{~m}$ maximal sprint test, maximal countermovement jump (CMJ), self-perceived muscle soreness and relaxation questionnaires, blood lactate, creatine kinase, testosterone, cortisol and catecholamine levels. The selfperceived feeling of relaxation after 60 minutes recovery was better $(p<0.05)$ after CWI and CWT than ACT and TWI. Statistically significant differences were not observed between the recovery methods in any other marker. In the $30 \mathrm{~m}$ sprint test, however, slower running time was found in ACT $(p<0.001)$ and CWT $(p=$ $0.005)$, and reduced CMJ results $(p<0.05)$ were found in ACT when the results were compared to baseline values. Based on these findings, it can be concluded that CWI and CWT improve the acute feeling of relaxation that can play a positive role in athletes' performance and well-being.
\end{abstract}

Key words: cryotherapy - recovery methods - hormones - feelings of relaxation 


\section{INTRODUCTION}

Athletes train intensively in an attempt to enhance their performance and induce adaptations. As a result of a single exercise session or training period, athletes may experience acute feelings of fatigue and decreases in physical performance. Fatigue in the body should be minimized by enhancing recovery and thus maximizing competition performance. Athletes are increasingly using various techniques to accelerate recovery (32). Athletic recovery modalities include active recovery, stretching, massage, anti-inflammatory drugs, compression garments, electrical stimulation, and the combinations of these recovery methods (3). In addition, water immersion methods are common among athletes, and they can be divided into four different categories: cold water immersion $\left(\mathrm{CWI} ; \leq 20^{\circ} \mathrm{C}\right)$, hot water immersion $\left(\mathrm{HWl} ; \geq 36^{\circ} \mathrm{C}\right)$, thermoneutral water immersion ( $\mathrm{TWl} ; 21-35^{\circ} \mathrm{C}$ ), and contrast water therapy (CWT; alternating $\mathrm{CWI}$ and $\mathrm{HWI})(32)$. After sufficient periods of recovery, athletes may enhance their performance due to a supercompensation effect occuring (12).

Water immersion techniques have been examined in the scientific literature (32), but the results of their effectiveness in enhancing recovery are conflicting $(2,6,8,10,11,16,23-26,28,32)$. Furthermore, the benefits of water immersion methods are often compared to passive recovery only $(6,8-11,16,21-23,25$, $28,30)$. There are also contrasting results regarding the benefits of active recovery methods compared to passive recovery (31). It appears that active recovery methods are largely ineffective for improving most psychophysiological markers of post-exercise recovery, but may nevertheless offer some benefits compared with passive recovery (31). In addition, athletes often perform an active form of recovery and upon completion of this, they may use other forms of recovery methods.

Compared to passive recovery and other water immersion methods, CWI has been found to be effective in improving the recovery of strength and power capacities after exercise protocols in several team sports and interval based endurance exercise $(2,8,16,21,22)$, but not after eccentric strength loadings $(11,24)$. After team sport exercise, the recovery of maximal and explosive strength and sprint performance was found to improve when the participants used CWI compared to passive recovery and other water immersion methods $(2,8,16)$. In turn, CWI was not found to promote the recovery of maximal voluntary contraction (MVC) after eccentric strength exercise compared to TWI (24) and passive recovery (11).

There are conflicting results regarding the effectiveness of CWT to the recovery of strength and power capacities $(10,16,28,30)$. For example, Vaile et al. $(28,30)$ found that CWT inhibited decreases in maximal and explosive strength performance compared to passive recovery. On the other hand, the recovery of maximal and explosive strength and sprint performance was not found to improve after CWT compared to passive recovery $(10,16)$. TWI was found to promote the recovery of explosive strength compared to passive recovery $(6,25)$. However, the aerobic movement in the water could have affected the recovery (32), and several studies have been shown that TWI did not enhance recovery $(5,26,27)$. In TWI only hydrostatic pressure acts on the body, while in the other water immersion methods the water temperature may act with the hydrostatic pressure (32).

There are inconsistent results concerning the benefits of water immersion methods on the markers of exercise induced muscle damage (EIMD). CWI was found to prevent EIMD after endurance and team sport exercise protocols compared to active and passive recovery and other water immersion methods $(2,8,16$, $21,22)$, but not after single-joint eccentric exercise. CWI was also found to prevent the increase of serum 
creatine kinase (CK) activity after endurance and team sport exercise protocols $(2,21)$. Furthermore, the increase of serum CK activity was not inhibited after eccentric strength exercise $(11,24)$. Contradictory findings have also been reported with regards to how CWT affects muscle soreness (MS) $(10,16,23,30)$. Vaile et al. (30) found that CWT decreased MS compared to passive recovery, while Ingram et al. (16) and Vaile et al. (28) did not find that CWT would affect the appearance of MS compared to passive recovery. Furthermore, CWT was not found to prevent leakage of CK $(10,16,23,28,30)$, while TWI was found to not prevent MS and leakage of CK from muscles $(2,23)$.

There are studies that have investigated effects of CWI on hormonal responses. CWI was found to increase acute serum cortisol concentrations immediately or 30 min post water immersion $(14,17)$ but after an hour of immersion, they had returned to baseline levels (14). In turn, Lindsay et al. (20) found a decrease in percentage change of serum cortisol concentrations two hours after $\mathrm{CWI}$ compared to the passive recovery group. Halson et al. (13), however, did not find a difference in cortisol concentration between CWI and passive recovery $40 \mathrm{~min}$ after immersion. In addition, there were no differences in serum testosterone and plasma epinephrine and norepinephrine concentrations between CWI and passive recovery at 40-60 min after immersion $(13,21)$. The purpose of the present study was to determine the effectiveness of three water immersion interventions performed after active recovery in comparison to active recovery alone on physical and mental performance. In addition, several physiological responses were monitored. The recovery protocol was designed to match with possible recovery routines used by athletes. Thus, the water immersion interventions were performed right after a period of active recovery. 


\section{METHODS}

\section{Experimental Approach to the Problem}

Despite the popularity of implementing water immersion methods among athletes, there is limited evidence to suggest the effectiveness of these methods on subsequent athletic performance. According to the literature, a bout of eccentric exercise may induce muscle soreness and exercise-induced muscle damage (EIMD) (15). Therefore, a repeated measures approach was used to examine the effects of four different recovery interventions on exercise performance following a short-term exercise protocol with maximal effort. Each subject performed all the recovery interventions that were included in the study. Study design was used to evaluate the effects of water immersion methods on physical performances, subjective ratings of MS and the feeling of relaxation, hormones and CK.

\section{Participants}

The participants $(n=9)$ were physically active men (mean \pm SD age $26 \pm 3.7 \mathrm{yrs}$, age range 20-35 yrs, body mass $78.6 \pm 11.6 \mathrm{~kg}$, height $1.81 \pm 0.09 \mathrm{~m}$, body fat $15.8 \pm 4.0 \%$ ). At the recruitment stage and before all the measurement period, the health status of the subjects was investigated via a health survey. After being informed about all of the details of the experimental procedures and methods including, the potential risks of the study, each participant signed an informed consent. The participants completed food and activity diaries to standardize nutrition and physical activity during the recovery period. Ethical approval for the study was obtained from the Ethical Committee of University of Jyväskylä, and the study was in accordance with the Declaration of Helsinki.

\section{Experimental designs and procedures}

A schematic representation of the protocol is provided in Figure 1. Participants were randomly allocated to active recovery only (ACT), a thermoneutral water immersion (TWI), cold water immersion (CWI) or contrast water therapy (CWT) groups. All participants were measured in four different experimental conditions (separated by at least 2 weeks), each time with a different recovery method. Prior to each recovery procedure, participants performed physical performance tests and a short-term exercise with maximal effort, which included jumping and sprinting. The recovery procedure included an active form of recovery (10 minutes bicycle ergometer, Monark 839 E, Vansbro, Sweden; heart rate 120-140 bpm, 60-73 \% from age-calculated maximum heart rate) whilst concurrently consuming a recovery beverage (Gainomax, $40 \mathrm{~g}$ carbohydrates and $20 \mathrm{~g}$ protein) and water immersion.

Hormones and biochemical markers of muscle damage were obtained at baseline, and at 5, 40, and 60 min of the end of the loading, and 24,48 , and $96 \mathrm{~h}$ after a loading protocol. Furthermore, neuromuscular function was measured at baseline and 24,48 , and $96 \mathrm{~h}$ after a loading protocol, and perceptual markers of muscle damage and mood state were obtained at the baseline and 1, 24, 48, and $96 \mathrm{~h}$ after a loading protocol. There were not any statistically significant differences at the 96 -h time point.

FIGURE 1 about here. 
The participants lived their normal life during every recovery period. The participants completed food and activity diaries in the first recovery period, for standardizing nutrition and physical activity during the recovery period. The diaries were returned at the second, third and fourth recovery period and the participants were instructed to eat and perform activities similarly as they did during the first recovery period.

\section{Immersions}

Each recovery group was immersed underwater in a sitting position to the level of the xiphoid process for $10 \mathrm{~min}$ in a dedicated bath, while the ACT group remained seated in an empty bath for $10 \mathrm{~min}$. CWI and TWI were continuously immersed in water temperatures of 10 and $24^{\circ} \mathrm{C}$, respectively. In CWT, the participants alternated immersion at 38 and $10^{\circ} \mathrm{C}$ with five cycles of $1 \mathrm{~min}$ in each bath.

\section{Performance tests and the exercise protocol}

Performance tests included $30 \mathrm{~m}$ maximal sprint (Intraclass correlation coefficient, ICC $=0.97$ ), isometric leg press, and countermovement jump (CMJ; ICC $=0.95)$. The results of maximal isometric leg press are not presented in this study. In $30 \mathrm{~m}$ sprint test, the participants ran $30 \mathrm{~m}$ maximally and the time of the last 20 $\mathrm{m}$ was recorded with photocells (Srin Test Oy, Tallinn, Estonia). The participants had three attempts to achieve their best running performance with 3 min of rest between trials.

The flight time in CMJ height was used as a measure of maximal muscle power and was measured on a contact mat (University of Jyväskylä, Finland). The jump height (h) of CMJ was calculated from the flight time $(\mathrm{t})$ by formula: $\mathrm{h}=\mathrm{t}^{2} * \mathrm{~g} / 8$, where $\mathrm{g}=9.81 \mathrm{~m} / \mathrm{s}^{2}(4)$. The participants were asked to keep their hands on their hips and jump as high as possible. They had three attempts to achieve their best performance with 1 min of rest between trials. A self-determined range of motion was permitted.

The participants performed a short-term exercise protocol with maximal effort (total duration $45 \mathrm{~min}$ ), which included:

1) $2 \times 5 \times$ ten unilateral long jumps (walking back between repetitions / $5 \mathrm{~min}$ of rest between sets)

2) $2 \times 3 \times 60$ m running (the first set: $95 \%$ of maximum speed / the second set: $98 \%$ of maximum speed; 2 min of rest between repetitions / 5 min of rest between sets), and

3) $2 \times 200 \mathrm{~m}$ run at maximum speed ( 5 min of rest between).

The rest between exercises was $5 \mathrm{~min}$. During the exercise protocol, heart rate was continuously recorded with a heart rate monitor (Polar V800, Kempele, Finland).

\section{Collection and analysis of blood samples}

Blood lactate was measured to evaluate the metabolic state of the body induced by the exercise bouts. Blood samples were obtained from the fingertip and collected into capillary tubes $(20 \mu \mathrm{L})$, which were placed in a 1-mL haemolyzing solution and analysed automatically after the completion of testing according to the instructions of the manufacturer (EKF diagnostic, C-line system, Biosen, Germany).

To assess the immediate and prolonged (up to $96 \mathrm{~h}$ ) recovery following the exercise protocols, blood samples were drawn from the antecubital vein into EDTA tubes (Venosafe, Terumo, Belgium) at baseline 
and at 5, 40, and $60 \mathrm{~min}$ after the end of the loading, and 24, 48, and $96 \mathrm{~h}$ after the loading protocol. The baseline samples were collected in a fasted state and after breakfast. Furthermore, the 24,48 , and 96-h samples were collected at fasted state. In order to control the accuracy of the measurements, the hydration of the participants was regulated during the measurements. The participants were instructed to drink $150 \mathrm{ml}$ portion of water when they arrived, during the breakfast, after the warm-up, and before, during and immediately after the exercise protocol.

Haemoglobin and haematocrit were determined with Sysmex KX-21N (TOA Medical Electronics Co., Ltd., Kobe, Japan), and plasma volume change was determined after exercise from haemoglobin and haematocrit concentrations using the equation by Dill and Costill (7). However, the results from the corrected values by the plasma volume change did not differ from the original results. Thus, the values without plasma volume change are used in the present study.

The serum samples were held for $30 \mathrm{~min}$ at room temperature before being centrifuged for $10 \mathrm{~min}$ at 2000×g (Megafuge 1.0 R, Heraeus, Germany). The serum was kept at $-80^{\circ} \mathrm{C}$ until analysed.

Serum creatine kinase (CK) activity, cortisol (COR), and testosterone (TES) concentrations were analysed by using the Immulite 2000 and immunoassay kits (Immulite, Siemens, IL, USA). The detection limits and interassay coefficients of variation were $5.9 \%$ for $\mathrm{CK}, 5.5 \mathrm{nmol} / \mathrm{l}$ and $7.9 \%$ for $\mathrm{COR}$, and $1.5 \mathrm{pg} / \mathrm{ml}$ and $2.8 \%$ for testosterone, respectively.

The plasma samples were held for $30 \mathrm{~min}$ at a room temperature before being centrifuged for $10 \mathrm{~min}$ at $2000 \times g$ (Megafuge 1.0 R, Heraeus, Germany). The plasma was kept at $-80^{\circ} \mathrm{C}$ until analysed. Epinephrine and nonepinephrine in plasma samples were analysed by using the Dynex DS2 (Washington, USA). Sample analysis was based on an Enzyme-linked Immunosorbent Assay (ELISA) method. The analytical sensitivity was $8 \mathrm{pg} / \mathrm{ml}$ for epinephrine and $20 \mathrm{pg} / \mathrm{ml}$ for norepinephrine.

\section{Muscle soreness and mood states}

Muscle soreness (MS) of the legs were subjectively evaluated in each participant. The participants filled out the questionnaire evaluating MS before the exercise, one hour afterwards, and after 24, 48, and 96 hours recovery. MS were evaluated on a scale from zero to five $(0=$ no muscle pain, $5=$ very hard muscle soreness).

The participants filled out the profile of mood state (POMS) questionnaire at the same time points as the MS questionnaire. The questionnaire had 65 emotions, the magnitude of which was estimated from one to five ( $1=$ not at all, $5=$ very high). The results of the POMS questionnaire are not processed and analysed in this work, except the feelings of relaxation.

\section{Statistical Analyses}

The results were analysed using IBM SPSS Statistics 22.0 (IBM Corp. Armonk, NY, USA) and Microsoft Excel 2010 (Microsoft Corp. Redmond, USA). The variance analysis (ANOVA) of repeated measurements (Bonferroni) and non-parametric Friedman and Wilcoxon tests were used in the statistical analysis. Data are presented as means \pm SDs. Statistical significance was set at $p<0.05$. In addition, 95 or $90 \%$ confidence 
intervals $(\mathrm{Cl})$ for selected differences between means were calculated and are presented where appropriate. Effect sizes (ES) were calculated to determine meaningful differences. Magnitudes of difference were classed as small $(<0.5)$, moderate $(0.5-0.8)$ and large $(>0.8)$. Only the large ES are presented in the text. The test-retest reliability was tested for independent variables with intraclass correlation coefficient (ICC). 


\section{RESULTS}

\section{Performance measurements}

In both the $30 \mathrm{~m}$ sprint test and CMJ, no significant differences were found between the recovery methods $(p=0.078-0.934)$. Although, the large ES value was found in the $30 \mathrm{~m}$ sprint test after $24 \mathrm{~h}$ recovery between TWI and ACT (1.32; $p=0.078)$. The $95 \% \mathrm{Cl}$ was -3.69 to 7.37 between these two recovery methods. After $48 \mathrm{~h}$ recovery, the large ES value was found between TWI and CWT (1.12; $p=0.149)$. The 95 $\% \mathrm{Cl}$ was -0.58 to 4.46 between these two recovery methods. Furthermore, the large ES value was found in CMJ after $24 \mathrm{~h}$ recovery between TWI and ACT (0.85). The $95 \% \mathrm{Cl}$ was -9.62 to 0.90 between these two recovery methods.

When the results of the $30 \mathrm{~m}$ sprint test were compared to the baseline values within the recovery method, a slower running time was found after 24 hours recovery in ACT ( $p=0.001 ; 95 \% \mathrm{Cl}=0.05,0.13$ ) and CWT $(p=0.005 ; 95 \% \mathrm{Cl}=0.03,0.12)$. Similarly after 48 hours recovery, the running time was slower after CWT $(p=0.023 ; 95 \% \mathrm{Cl}=0.02,0.19$ ), when the results were compared to the baseline values. When the results of $\mathrm{CMJ}$ were compared to the baseline values within the recovery method, reduced $\mathrm{CMJ}$ results were found after 24 hours recovery in ACT ( $p=0.033 ; 95 \% \mathrm{Cl}=-3.88,-0.18)$ (Figures 2 and 3 ).

FIGURE 2 about here.

FIGURE 3 about here.

\section{Cortisol, testosterone, and catecholamines}

There were no significant differences between recovery methods in the values of cortisol, testosterone, epinephrine, and norepinephrine $(p=0.081-1.000)$. When the values were compared within the recovery methods, the serum testosterone concentration was found to be lower (CWI: $p=0.007,95 \% \mathrm{Cl}=-6.95$, 1.23; TWI: $p=0.001,95 \% \mathrm{Cl}=-6.08,-1.08$; CWT: $p=0.001,95 \% \mathrm{Cl}=-6.08,-1.97)$ than the baseline value after one-hour recovery after all the water immersion methods. After CWI higher epinephrine $(p=0.012$, $95 \% \mathrm{Cl}=-0.02,0.26)$ and norepinephrine concentrations $(\mathrm{p}=0.013,95 \% \mathrm{Cl}=0.14,0.79)$ were observed after 40 minutes recovery when the results were analysed within recovery procedures. (Table 1 ).

TABLE 1 about here.

\section{Muscle soreness and creatine kinase (CK) activity}

In self-perceived muscle soreness no significant differences were found between the recovery methods $(p=$ 0.272-0.963) (Table 2). When the results were compared to the baseline values within the recovery method, higher MS was found after 24 (ACT: $p=0.018,95 \% \mathrm{Cl}=0.75,3.00 ; \mathrm{CWI}: \mathrm{p}=0.031,95 \% \mathrm{Cl}=0$, 2.25; TWI: $p=0.015,95 \% \mathrm{Cl}=0,1 ; \mathrm{CWT}: \mathrm{p}=0.014,95 \% \mathrm{Cl}=1,3)$ and $48(\mathrm{p}=0.011-0.026 ; A C T: p=0.011$, $95 \% \mathrm{Cl}=1.25,3$; CWI: $p=0.017,95 \% \mathrm{Cl}=1,3.25$; TWI: $p=0.011,95 \% \mathrm{Cl}=1.5,3.5 ;$ CWT: $p=0.026,95 \%$ $\mathrm{Cl}=1,5$ ) hours recovery in all recovery methods. Furthermore, after 60 minutes recovery the muscle soreness was significantly higher $(p=0.039,90 \% \mathrm{Cl}=1,3)$ in the active recovery group when the results were compared to the baseline values. 
There were no significant differences between recovery methods in the CK activity $(p=0.334-0.615)$ (Table 2). When the results were compared to the baseline values within the recovery method, higher $C K$ activities were found after 24 (ACT: $p=0.003,95 \% \mathrm{Cl}=0.78,1.94$; CWI: $p=0.014,95 \% \mathrm{Cl}=0.71,2.19$; TWI: $p=$ $0.014,95 \% \mathrm{Cl}=0.49,1.50$; CWT: $\mathrm{p}=0.005,95 \% \mathrm{Cl}=0.22,2.19$ ) and 48 hours (ACT: $\mathrm{p}=0.012,95 \% \mathrm{Cl}=$ 0.64, 1.78; CWI: $p=0.042,95 \% \mathrm{Cl}=0.48,1.79$; TWI: $p=0.009,95 \% \mathrm{Cl}=0.42,1.19 ; \mathrm{CWT}: \mathrm{p}=0.028,95 \% \mathrm{Cl}$ $=0.18,2.69$ ) recovery in all recovery methods. The values of muscle soreness and logarithm corrected values of CK activity are presented in Table 2.

TABLE 2 about here.

\section{Blood lactate and heart rate}

Blood lactate at 5 and 40 minutes after the exercise protocol and heart rate (HR) values during the exercise protocol are presented in Table 3. No differences between the recovery methods in blood lactate $(p=$ $0.243-1.000)$ and heart rate $(p=0.575-0.833)$ were observed. The ICC values for average HR, maximum HR and lactate 5 minutes after exercise were not different across the exercise protocols with ICC values of $R=$ $0.91,0.95$, and 0.86 , respectively.

TABLE 3 about here.

\section{The self-perceived feeling of relaxation}

The self-perceived feeling of relaxation after one-hour recovery was better after $\mathrm{CWI}(\mathrm{p}=0.025,90 \% \mathrm{Cl}=-$ $1,-1)$ and CWT $(p=0.034,90 \% \mathrm{Cl}=1,2)$ than the active recovery and TWI. There were no significant differences between recovery methods after $24 \mathrm{~h}$ recovery. Furthermore, the large ES values were found between ACT and CWI (1.05), and TWI and CWT (0.94) after 60 min recovery.

FIGURE 4 about here. 


\section{DISCUSSION}

The main finding of this study was that the self-perceived feeling of relaxation after 60 minutes recovery was statistically significantly higher after CWI and CWT than ACT and TWI, respectively. Significant differences were not observed between the recovery methods in any other marker.

Based on the present study, CWI and CWT improve the acute feeling of relaxation. This can play a positive role in performance and well-being of the athletes. In a previous study, the subjects reported that thermoneutral water exercise was more effective and a more appreciated recovery intervention than sitting at rest (5). Furthermore, it is accepted that psychological effects are inherently linked to the physiological effects, and can be of major importance for performance $(26,31)$. Thus, both physiological and psychological factors should be taken into account in the development of physical performance and recovering from exercises. Furthermore, individualising the recovery, personal preference should be taken into consideration.

In self-perceived muscle soreness, no statistically significant differences were found between the recovery methods. Similarly, in a study by Pournot et al. (23), the authors did not find any difference between CWI, TWI, CWT, and passive recovery. However, it is important to acknowledge that in some individuals, water immersion methods reduced acute muscle soreness (after 60 min recovery) when the results were analysed within recovery methods. CWI has been found to prevent muscle soreness after endurance and team sport exercise protocols compared to active and passive recovery and other water immersion techniques $(2,8,16,18,20-22)$. CWI is also believed to reduce MS by inhibiting acute inflammation (9). However, this was not found after a single-joint eccentric exercise $(11,24)$. Furthermore, there are conflicting results about how CWT affects muscle soreness $(10,28,30)$.

In serum CK concentration, no statistically significant differences were found between the recovery methods. In previous studies, CWI has been found to prevent increases of serum or plasma CK $(2,21)$. This was not found after a single-joint eccentric exercise $(11,24)$. Furthermore, CWT has not been found to affect leakage of CK $(10,28,30)$, and TWI has not either been found to affect the leakage of CK compared to $\mathrm{CWI}$ and passive recovery $(2,23)$. Sellwood et al. $(24)$ used similar temperature as in this study $\left(24^{\circ} \mathrm{C}\right)$ in TWI, and they did not find any difference between TWI and CWI.

In some individuals, the recovery of power and speed capacities can be improved by using CWI and TWI. This was found when the results were analysed within the recovery methods. On a group level, however, the change in physical performance variables were not significant. Thus, strong evidence of the benefit of water immersion methods to the recovery of the power capacities was not obtained. On the other hand, the large effect sizes found between the recovery methods would support the benefits of TWI for improved recovery of power and speed capacities. Similarly, Pournot et al. (23) did not find differences between CWI, TWI, CWT, and passive recovery, but they found that CWI could improve the recovery of power capacities when the results were compared to baseline within the recovery method. According to the literature, CWI has been found to be effective in recovery of strength and power capacities compered to TWI, CWT, and passive recovery $(2,8,33)$ However, there are also conflicting results(18). TWI was found to promote the recovery of explosive strength $(6,25)$, but again, there are contrasting findings $(5,26,27)$. It has been found that a water temperature of $15{ }^{\circ} \mathrm{C}$ could be better for improving maximal muscle power recovery than $5{ }^{\circ} \mathrm{C}$ 
(33) while no differences between the conditions of $10^{\circ} \mathrm{C}, 15^{\circ} \mathrm{C}$, and $20^{\circ} \mathrm{C}$ were observed (29). In the present study, water temperatures of 10 and $24^{\circ} \mathrm{C}$ were used, and it appears that there were no differences between these temperatures when considering the recovery of maximal power capacities.

There were no statistically significant differences in the concentrations of cortisol, testosterone, epinephrine, and norepinephrine between the recovery methods. In some individuals, the water immersion methods were shown to inhibit the recovery of the testosterone concentration, when the results were analysed within each recovery method. Furthermore, after CWI, higher epinephrine and norepinephrine levels were observed after 40 minutes recovery, when the results were analysed within each recovery procedure. This could indicate that CWI could acutely increase concentration of catecholamine and stress state of the body. However, 20 minutes later, their increase was not statistically significant anymore. Consequently, the effect of CWI on catecholamine concentration appears to be short-lived.

Previous studies have reported that CWI increases cortisol concentration compared to basal concentration ( $\leq 30 \mathrm{~min}$ after immersion) $(14,17)$. There is also research that has reported contrasting findings, where cortisol levels were lower following CWI than after passive recovery $(20,21)$ and did not change compared to basal levels (30 min after CWI/PAS) (21). In addition, it was found that after passive recovery, serum testosterone was statistically significantly higher after 60 minutes and 24 hours recovery compared to baseline concentration, but after CWI there were no statistically significant differences between testosterone concentrations in different time points (21). After exercise, the testosterone concentration decreased indicating an acute catabolic phase. Thereafter, the testosterone concentration has been found to recover back to the resting levels or even higher than resting levels, which indicated, an anabolic phase (19). The role of testosterone is important for physiological adaptations to exercise and training. The effects of testosterone include stimulation of protein synthesis and inhibition of protein degradation. Thus, the combination of these effects may promote muscle hypertrophy (34). The results of this study and the current literature $(13,21)$ indicate that CWI could inhibit the beginning of the anabolic state of the body. Furthermore, it was found that after 40 min recovery the cortisol concentration increased and the testosterone and epinephrine concentrations decreased compared baseline levels (13), which support the present study, which did not find any differences between CWI and passive recovery. In addition, it was found that CWI acutely increases norepinephrine concentration (17). This suggests that there is some evidence that water immersion methods may inhibit the beginning of the anabolic state. Furthermore, there is evidence that CWI could acutely increase epinephrine and norepinephrine concentrations and increase the stress response.

In conclusion, CWI and CWT improve the acute feeling of relaxation and this could play a positive role in performance and well-being of athletes. Furthermore, in some individuals, the recovery of power and speed capacities can be improved by using water immersion methods. Additionally, there is some evidence that water immersion methods may acutely inhibit the increase in testosterone concentration after exercise, and CWI could acutely increase epinephrine and norepinephrine concentration. It must be taken into account, that in this study the participants were physically active men, but not athletes. It has been noticed that physiological responses may differ between athletes and active men (1).

\section{Practical Applications}

According to the results of this study, it appears that water immersion methods as a recovery strategy should be used with caution. The acute feeling of relaxation is important and can be achieved within quite 
short time periods, which should consider when the time between competitions or matches is short. The results of this study shows that water immersion methods do not give a significant additional benefit to the recovery routines used by athletes when considering the recovery of physical performance and muscle cell damage. Further studies should focus on investigating the possible benefits of water immersion methods, both in terms of enhancing physical performance, recovery, muscle cell damage, and hormone function. It would also be beneficial to determine suitable water temperatures and the duration of immersion times. Furthermore, the understanding about the use of water immersion methods for different kinds of training and sports disciplines would offer a great deal for practical applications. Current evidence appears to show that water immersion methods may have some acute effects relating to hormonal function. However, the long-term effects of hormonal function on physiological variables and adaptations are an area that requires further investigation.

\section{Acknowledgements}

The present research was supported by Ecomarine Oy and Avantopool Oy. 


\section{References}

1. Ahtiainen JP, Pakarinen A, Alen M, Kraemer WJ, Hakkinen K. Muscle hypertrophy, hormonal adaptations and strength development during strength training in strength-trained and untrained men. Eur J Appl Physiol. 89(6):555-63, 2003.

2. Ascensao A, Leite M, Rebelo AN, Magalhaes S, Magalhaes J. Effects of cold water immersion on the recovery of physical performance and muscle damage following a one-off soccer match. J Sports Sci 29(3): 217-225, 2011.

3. Barnett A. Using recovery modalities between training sessions in elite athletes: Does it help? Sports Medicine 36(9): 781-796, 2006.

4. Bosco C, Luhtanen P, Komi PV. A simple method for measurement of mechanical power in jumping. European Journal of Applied Physiology \& Occupational Physiology 50(2): 273-282, 1983.

5. Cortis C, Tessitore A, D'Artibale E, Meeusen R, Capranica L. Effects of post-exercise recovery interventions on physiological, psychological, and performance parameters. Int J Sports Med 31(5): 327$335,2010$.

6. Dawson B, Cow S, Modra S, Bishop D, Stewart G. Effects of immediate post-game recovery procedures on muscle soreness, power and flexiblity levels over the next 48 hours. Journal of Science \& Medicine in Sport 8(2): 210-221, 2005.

7. Dill DB, Costill DL. Calculation of percentage changes in volumes of blood, plasma, and red cells in dehydration. J Appl Physiol 37(2): 247-248, 1974.

8. Elias GP, Wyckelsma VL, Varley MC, McKenna MJ, Aughey RJ. Effectiveness of water immersion on postmatch recovery in elite professional footballers. International journal of sports physiology \& performance 8(3): 243-253, 2013.

9. Eston R, Peters D. Effects of cold water immersion on the symptoms of exercise-induced muscle damage. Journal of Sports Sciences 17(3): 231-238, 1999.

10. French DN, Thompson KG, Garland SW, Barnes CA, Portas MD, Hood PE, Wilkes G. The effects of contrast bathing and compression therapy on muscular performance. Medicine \& Science in Sports \& Exercise 40(7): 1297-1306, 2008.

11. Goodall S, Howatson G. The effects of multiple cold water immersions on indices of muscle damage. Journal of Sports Science \& Medicine 7(2): 235-241, 2008.

12. Halson SL, Jeukendrup AE. Does overtraining exist?: An analysis of overreaching and overtraining research. Sports Medicine 34(14): 967-981, 2004.

13. Halson SL, Quod MJ, Martin DT, Gardner AS, Ebert TR, Laursen PB. Physiological responses to cold water immersion following cycling in the heat. International journal of sports physiology \& performance 3(3): 331346, 2008.

14. Hermanussen $M$, Jensen $F$, Hirsch N, Friedel K, Kroger B, Lang R, Just S, Ulmer J, Schaff $M$, Ahnert P. Acute and chronic effects of winter swimming on $\mathrm{LH}, \mathrm{FSH}$, prolactin, growth hormone, TSH, cortisol, serum glucose and insulin. Arctic Med Res 54(1): 45-51, 1995. 
15. Howatson G, van Someren KA. The prevention and treatment of exercise-induced muscle damage. Sports Medicine 38(6): 483-503, 2008.

16. Ingram J, Dawson B, Goodman C, Wallman K, Beilby J. Effect of water immersion methods on postexercise recovery from simulated team sport exercise. Journal of Science and Medicine in Sport 12(3): 417421, 2009.

17. Kauppinen K, Pajari-Backas M, Volin P, Vakkuri O. Some endocrine responses to sauna, shower and ice water immersion. Arctic Med Res 48(3): 131-139, 1989.

18. King M, Duffield R. The effects of recovery interventions on consecutive days of intermittent sprint exercise. Journal of Strength and Conditioning Research 23(6): 1795-1802, 2009.

19. Lac G, Berthon P. Changes in cortisol and testosterone levels and $\mathrm{T} / \mathrm{C}$ ratio during an endurance competition and recovery. / changement de niveau du cortisol de la testosterone et du rapport T/C lors d ' une competition d ' endurance et de la recuperation consecutive. Journal of Sports Medicine \& Physical Fitness 40(2): 139-144, 2000.

20. Lindsay A, Carr S, Cross S, Petersen C, Lewis JG, Gieseg SP. The physiological response to cold-water immersion following a mixed martial arts training session. Applied Physiology, Nutrition, \& Metabolism = Physiologie Appliquee, Nutrition et Metabolisme. 42(5):529-36, 2017.

21. Minett GM, Duffield R, Billaut F, Cannon J, Portus MR, Marino FE. Cold-water immersion decreases cerebral oxygenation but improves recovery after intermittent-sprint exercise in the heat. Scandinavian Journal of Medicine \& Science in Sports 24(4): 656-666, 2014.

22. Pointon M, Duffield R, Cannon J, Marino FE. Cold water immersion recovery following intermittentsprint exercise in the heat. European journal of applied physiology 112(7): 2483-2494, 2012.

23. Pournot H, Bieuzen F, Duffield R, Lepretre $P$, Cozzolino C, Hausswirth C. Short term effects of various water immersions on recovery from exhaustive intermittent exercise. Eur J Appl Physiol 111(7): 1287-1295, 2011.

24. Sellwood KL, Brukner P, Williams D, Nicol A, Hinman R. Ice-water immersion and delayed-onset muscle soreness: A randomised controlled trial. Br J Sports Med 41(6): 392-397, 2007.

25. Takahashi J, Ishihara K, Aoki J. Effect of aqua exercise on recovery of lower limb muscles after downhill running. J Sports Sci 24(8): 835-842, 2006.

26. Tessitore A, Meeusen R, Cortis C, Capranica L. Effects of different recovery interventions on anaerobic performances following preseason soccer training. Journal of Strength \& Conditioning Research 21(3): 745750, 2007.

27. Tessitore A, Meeusen R, Pagano R, Benvenuti C, Tiberi M, Capranica L. Effectiveness of active versus passive recovery strategies after futsal games. Journal of Strength \& Conditioning Research 22(5): 14021412, 2008.

28. Vaile J, Gill N, Blazevich A. The effect of contrast water therapy on symptoms of delayed onset muscle soreness. Journal of Strength and Conditioning Research 21(3): 697-702, 2007. 
29. Vaile J, Halson S, Gill N, Dawson B. Effect of cold water immersion on repeat cycling performance and thermoregulation in the heat. Journal of Sports Sciences 26(5): 431-440, 2008.

30. Vaile J, Halson S, Gill N, Dawson B. Effect of hydrotherapy on the signs and symptoms of delayed onset muscle soreness. Eur J Appl Physiol 102(4): 447-455, 2008.

31. Van Hooren B, Peake JM. Do we need a cool-down after exercise? A narrative review of the psychophysiological effects and the effects on performance, injuries and the long-term adaptive response. Sports Medicine. 2018. https://dx-doi-org.ezproxy.jyu.fi/10.1007/s40279-018-0916-2

32. Versey N, Halson S, Dawson B. Water immersion recovery for athletes: Effect on exercise performance and practical recommendations. Sports Med 43(11): 1101-1130, 2013.

33. Vieira A, Siqueira AF, Ferreira-Junior JB, do Carmo J, Durigan JL, Blazevich A, Bottaro M. The effect of water temperature during cold-water immersion on recovery from exercise-induced muscle damage. Int $J$ Sports Med 37(12): 937-943, 2016.

34. Vingren JL, Kraemer WJ, Ratamess NA, Anderson JM, Volek JS, Maresh CM. Testosterone physiology in resistance exercise and training: The up-stream regulatory elements. Sports Medicine 40(12): 1037-1053, 2010. 
Table 1. Mean $( \pm S D)$ serum cortisol and testosterone levels and plasma epinephrine and norepinephrine concentrations.

\begin{tabular}{|c|c|c|c|c|c|c|c|}
\hline & & Fasting & Pre & Post 5min & $\begin{array}{l}\text { Post } \\
40 \mathrm{~min}\end{array}$ & Post 60min & Post 24h \\
\hline \multirow{4}{*}{$\begin{array}{l}\text { Cortisol } \\
\text { (nmol/l) }\end{array}$} & $\overline{A C T}$ & $547 \pm 108$ & $469 \pm 89$ & $444 \pm 190$ & $469 \pm 184$ & $409 \pm 150$ & $474 \pm 94$ \\
\hline & CWI & $451 \pm 67$ & $431 \pm 50$ & $394 \pm 153$ & $458 \pm 129$ & $395 \pm 132$ & $434 \pm 88$ \\
\hline & TWI & $458 \pm 97$ & $482 \pm 116$ & $430 \pm 136$ & $448 \pm 161$ & $391 \pm 138$ & $451 \pm 99$ \\
\hline & CWT & $488 \pm 81$ & $486 \pm 82$ & $420 \pm 146$ & $410 \pm 110$ & $357 \pm 12$ & $486 \pm 97$ \\
\hline \multirow{4}{*}{$\begin{array}{l}\text { Testosterone } \\
\text { (nmol/l) }\end{array}$} & $\mathrm{ACT}$ & $17.3 \pm 4.4$ & $14.5 \pm 3.6$ & $16.9 \pm 6.4$ & $14.4 \pm 5.6$ & $12.9 \pm 5.1$ & $17.0 \pm 3.4$ \\
\hline & CWI & $18.1 \pm 4.2$ & $15.6 \pm 2.9$ & $17.9 \pm 4.8$ & $14.7 \pm 4.5$ & $11.5 \pm 3.8 * *$ & $16.8 \pm 3.3$ \\
\hline & TWI & $18.8 \pm 3.3$ & $16.5 \pm 3.4$ & $18.0 \pm 4.0$ & $15.4 \pm 4.3$ & $12.6 \pm 3.2 * * *$ & $17.8 \pm 4.1$ \\
\hline & CWT & $17.7 \pm 3.4$ & $14.7 \pm 2.5$ & $15.5 \pm 2.8$ & $12.9 \pm 3.3$ & $10.7 \pm 2.6^{* * *}$ & $17.3 \pm 3.1$ \\
\hline \multirow[t]{2}{*}{ Epinephrine } & $\mathrm{ACT}$ & $0.34 \pm$ & $0.23 \pm$ & $0.48 \pm$ & $0.29 \pm$ & $0.24 \pm 0.13$ & $0.21 \pm$ \\
\hline & & 0.15 & 0.08 & $0.36 *$ & 0.13 & & $0.06 *$ \\
\hline \multirow[t]{6}{*}{ (ng/ml) } & CWI & $0.32 \pm$ & $0.21 \pm$ & $0.39 \pm$ & $0.34 \pm$ & $0.31 \pm 0.12$ & $0.18 \pm$ \\
\hline & & 0.19 & 0.10 & $0.13 * *$ & $0.17 *$ & & $0.10 * *$ \\
\hline & TWI & $0.25 \pm$ & $0.23 \pm$ & $0.44 \pm$ & $0.31 \pm$ & $0.22 \pm 0.14$ & $0.21 \pm$ \\
\hline & & 0.08 & 0.11 & $0.29 *$ & 0.19 & & 0.11 \\
\hline & CWT & $0.27 \pm$ & $0.24 \pm$ & $0.35 \pm$ & $0.36 \pm$ & $0.23 \pm 0.10$ & $0.19 \pm$ \\
\hline & & 0.15 & 0.15 & 0.18 & 0.14 & & 0.13 \\
\hline \multirow[t]{2}{*}{ Norepinephrine } & ACT & $1.10 \pm$ & $1.01 \pm$ & $3.05 \pm$ & $1.04 \pm$ & $1.05 \pm 0.48$ & $1.08 \pm$ \\
\hline & & 0.60 & 0.40 & $3.28 * *$ & 0.47 & & 0.49 \\
\hline \multirow[t]{6}{*}{ (ng/ml) } & CWI & $0.80 \pm$ & $0.83 \pm$ & $1.84 \pm$ & $1.28 \pm$ & $1.06 \pm 0.49$ & $0.56 \pm$ \\
\hline & & 0.22 & 0.33 & $0.76 *$ & $0.39 *$ & & 0.27 \\
\hline & TWI & $0.75 \pm$ & $0.79 \pm$ & $1.83 \pm$ & $0.79 \pm$ & $0.87 \pm 0.47$ & $0.76 \pm$ \\
\hline & & 0.24 & 0.27 & $1.14^{*}$ & 0.29 & & 0.37 \\
\hline & CWT & $0.92 \pm$ & $0.81 \pm$ & $1.54 \pm$ & $1.04 \pm$ & $0.80 \pm 0.41$ & $0.72 \pm$ \\
\hline & & 0.42 & 0.32 & $0.45 *$ & 0.35 & & 0.48 \\
\hline
\end{tabular}

$\mathrm{ACT}$ = active recovery, $\mathrm{CWI}=$ cold water immersion, $\mathrm{TWI}=$ thermoneutral water immersion, $\mathrm{CWT}=$ contrast water therapy, fasting $=$ the measurement before breakfast, pre $=$ measurement before exercise, post $=$ measurement after exercise. ${ }^{*} p<0.05$ difference compared to baseline values within recovery method, ${ }^{* *}$ $p<0.01$ difference compared to baseline values within recovery method, $* * * p<0.001$ difference compared to baseline values within recovery method. The acute changes $(5,40$, and $60 \mathrm{~min})$ are compared to pre-values and the long-term changes $(24 \mathrm{~h})$ are compared to fasting-values. 
Table 2 Mean $( \pm S D)$ muscle soreness and logarithm corrected values of creatine kinase activity.

\begin{tabular}{llllll}
\hline & & Pre & Post 60min & Post 24h & Post 48h \\
\hline MS (1-5) & ACT & $0.3 \pm 0.7$ & $1.2 \pm 1.0^{*}$ & $1.8 \pm 1.4^{*}$ & $2.3 \pm 1.4^{*}$ \\
& CWI & $0.1 \pm 0.3$ & $0.4 \pm 0.7$ & $1.3 \pm 1.1^{*}$ & $1.7 \pm 1.2^{*}$ \\
& TWI & $0.1 \pm 0.3$ & $0.4 \pm 0.5$ & $1.3 \pm 0.7^{*}$ & $2.3 \pm 1.2^{*}$ \\
& CWT & $0.4 \pm 0.5$ & $0.4 \pm 0.7$ & $1.8 \pm 1.1^{*}$ & $2.1 \pm 1.5^{*}$ \\
In CK (U/I) & ACT & $4.97 \pm 0.63$ & & $6.39 \pm 0.69^{* *}$ & $6.09 \pm 0.85^{*}$ \\
& CWI & $4.84 \pm 0.38$ & & $6.18 \pm 0.90^{*}$ & $5.86 \pm 0.80^{*}$ \\
& TWI & $5.05 \pm 0.52$ & & $6.04 \pm 0.89^{*}$ & $5.84 \pm 0.73^{* *}$ \\
& CWT & $5.43 \pm 0.74$ & & $6.61 \pm 0.64^{* *}$ & $6.66 \pm 0.90^{*}$ \\
\hline
\end{tabular}

$\mathrm{ACT}=$ active recovery, $\mathrm{CWI}=$ cold water immersion, $\mathrm{TWI}=$ thermoneutral water immersion, $\mathrm{CWT}=$ contrast water therapy, pre $=$ measurement before exercise, post $=$ measurement after exercise. ${ }^{*} p<0.05$ difference compared to the baseline values within recovery method, ${ }^{* *} p<0.01$ difference compared to baseline values within recovery method. 
Table 3 Mean $( \pm$ SD) blood lactate concentration after the exercises of 5 and 40 min and heart rate values during the exercises.

\begin{tabular}{|c|c|c|c|c|}
\hline & ACT & CWI & TWI & CWT \\
\hline $\begin{array}{l}\text { Lactate post } 5 \mathrm{~min} \\
(\mathrm{mmol} / \mathrm{l})\end{array}$ & $14.6 \pm 2.2$ & $14.9 \pm 3.2$ & $15.4 \pm 2.3$ & $14.5 \pm 3.2$ \\
\hline $\begin{array}{l}\text { Lactate post } 40 \mathrm{~min} \\
(\mathrm{mmol} / \mathrm{l})\end{array}$ & $4.4 \pm 1.1$ & $4.1 \pm 1.4$ & $4.8 \pm 3.6$ & $3.2 \pm 1.0$ \\
\hline Average HR (bpm) & $134 \pm 8$ & $134 \pm 8$ & $135 \pm 10$ & $135 \pm 12$ \\
\hline Maximum HR (bpm) & $181 \pm 7$ & $181 \pm 6$ & $183 \pm 7$ & $182 \pm 8$ \\
\hline
\end{tabular}

$\mathrm{ACT}=$ active recovery, $\mathrm{CWI}=$ cold water immersion, $\mathrm{TWI}=$ thermoneutral water immersion, $\mathrm{CWT}=$ contrast water therapy, post $=$ measurement after exercise. 


\section{Figure legends}

Figure 1. A schematic representation of the protocol. POMS = profile of mood state questionnaire, $\mathrm{MS}=$ muscle soreness questionnaire, $30 \mathrm{~m}=30$ meter running test, $\mathrm{MVC}=$ maximal voluntary contraction on isometric leg press, $\mathrm{CMJ}=$ countermovement jump, $\mathrm{ACT}=$ active recovery, $\mathrm{CWI}=$ cold water immersion, $\mathrm{TWI}=$ thermoneutral water immersion, $\mathrm{CWT}=$ contrast water therapy, $\mathrm{PRE}=$ before exercise protocol, POST $=$ after exercise protocol.

Figure 2. Mean $( \pm S D)$ relative changes in 30 meter running time. $A C T=$ active recovery, $C W I=$ cold water immersion, $\mathrm{TWI}=$ thermoneutral water immersion, $\mathrm{CWT}=$ contrast water therapy, pre = measurement before exercise, post $=$ measurement after exercise. ${ }^{*} p<0.05$ difference compared to baseline values within recovery method from absolute values, ${ }^{* *} p<0.005$ difference compared to baseline values within recovery method from absolute values, ${ }^{* * *} p<0.001$ difference compared to baseline values within recovery method from absolute values.

Figure 3. Mean $( \pm S D)$ relative change in countermovement jump. $A C T=$ active recovery, $C W I=$ cold water immersion, $\mathrm{TWI}=$ thermoneutral water immersion, $\mathrm{CWT}=$ contrast water therapy, pre $=$ measurement before exercise, post $=$ measurement after exercise, $\mathrm{CMJ}=$ countermovement jump. $* \mathrm{p}<0.05$ difference compared to baseline values within recovery method from absolute values.

Figure 4. Mean $( \pm S D)$ absolute values of the self-perceived feeling of relaxation. $A C T=$ active recovery, $C W I$ = cold water immersion, $\mathrm{TWI}=$ thermoneutral water immersion, $\mathrm{CWT}=$ contrast water therapy, pre $=$ measurement before the exercise, post $=$ measurement after the exercise. $\# p<0.05$ difference between recovery methods. 
Figure 1.

\section{EXPERIMENTAL DESIGN}

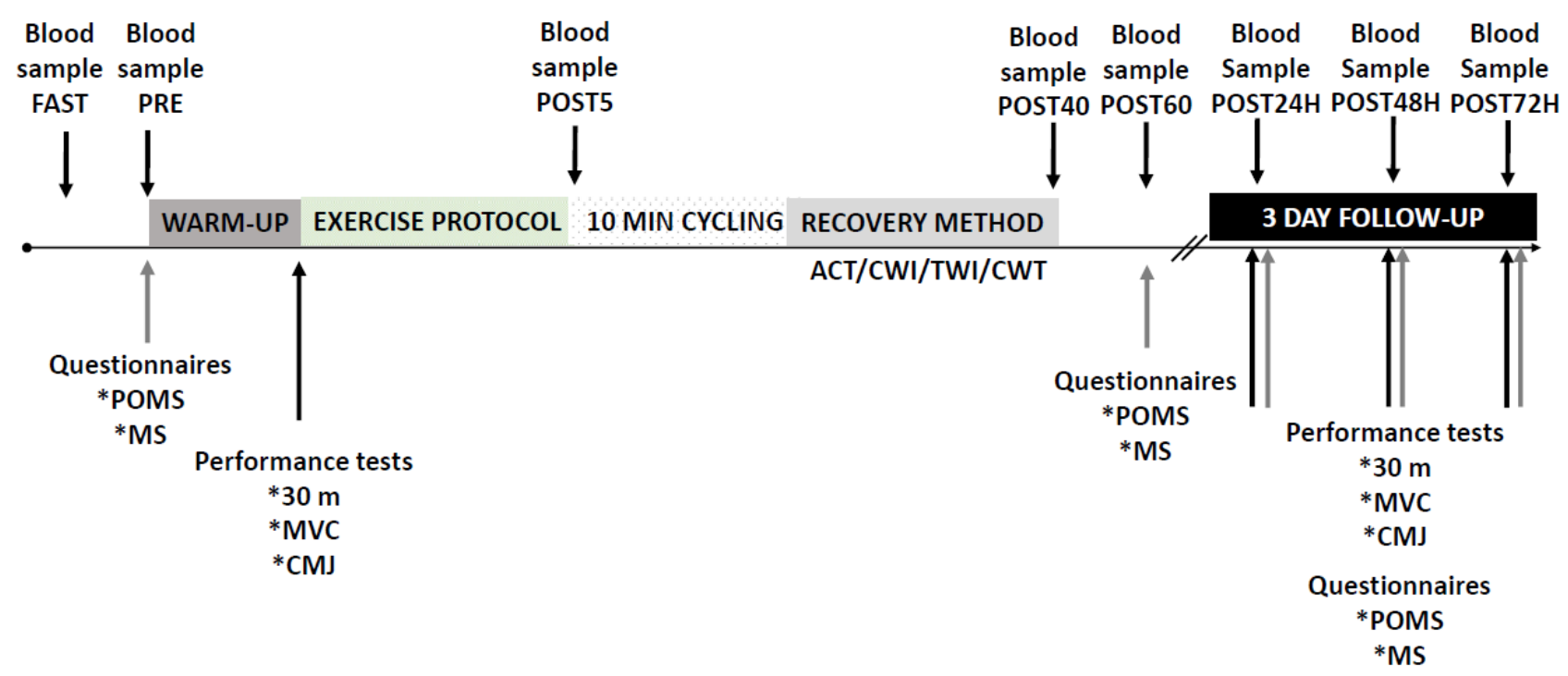


Figure 2.

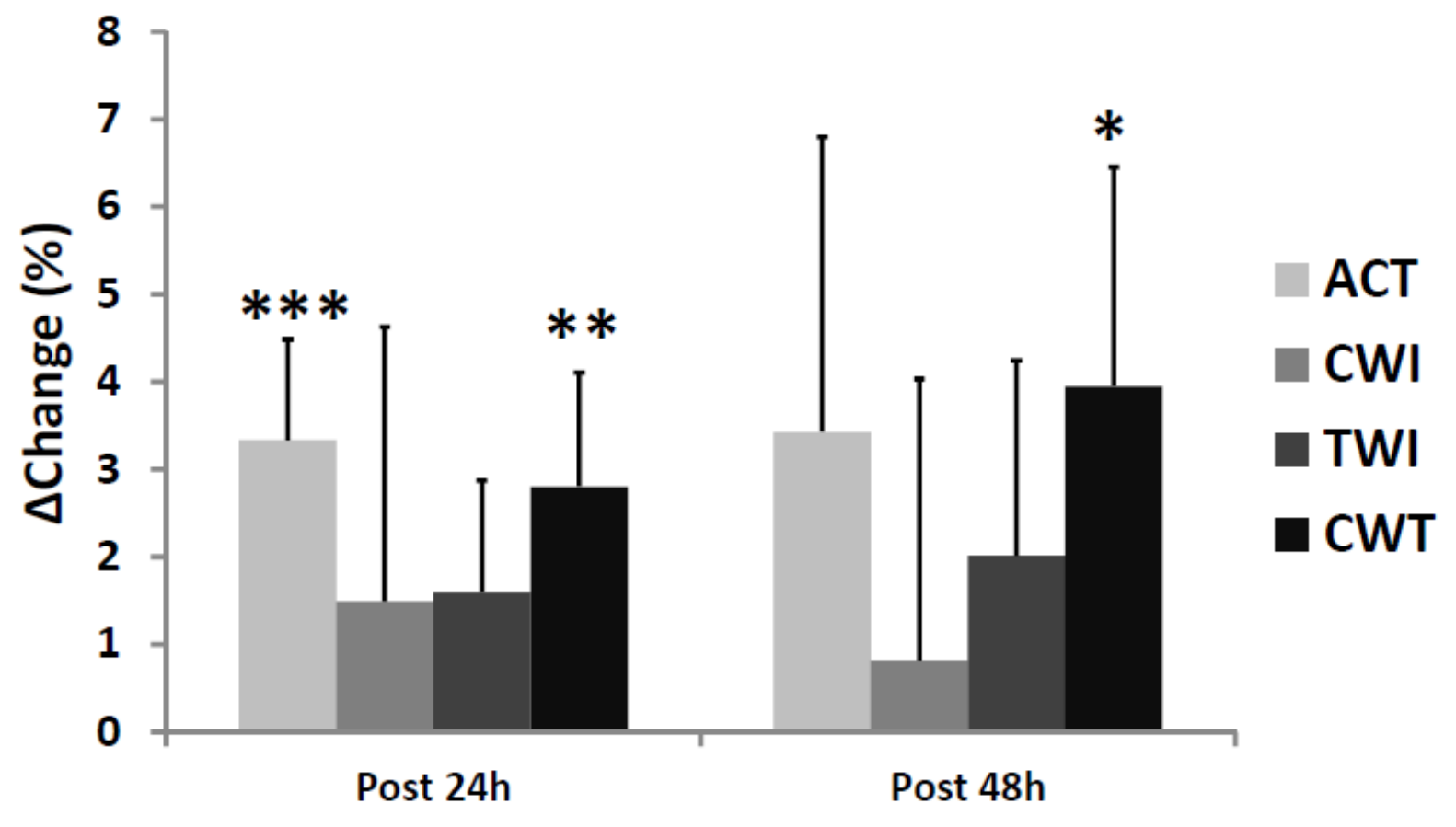


Figure 3.

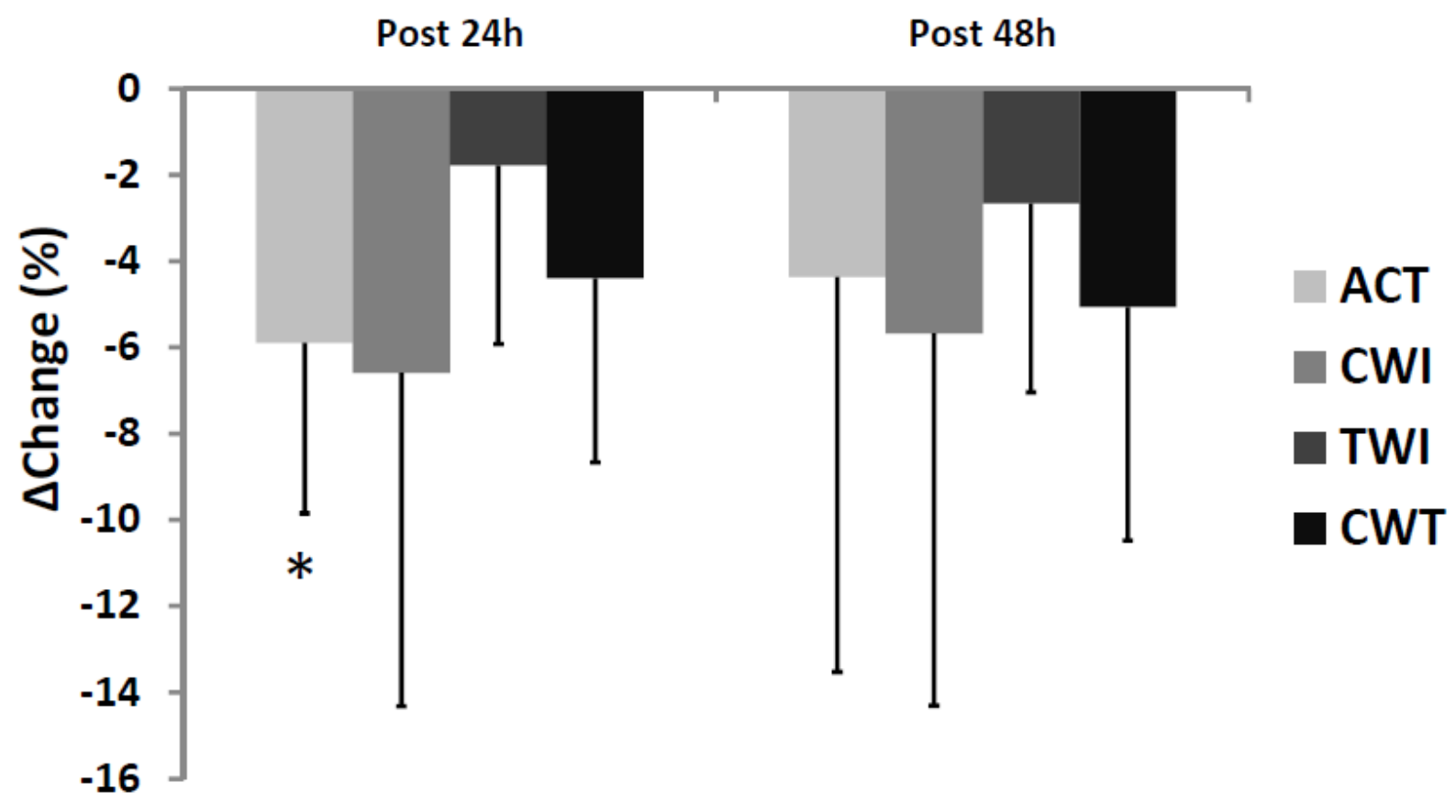


Figure 4.

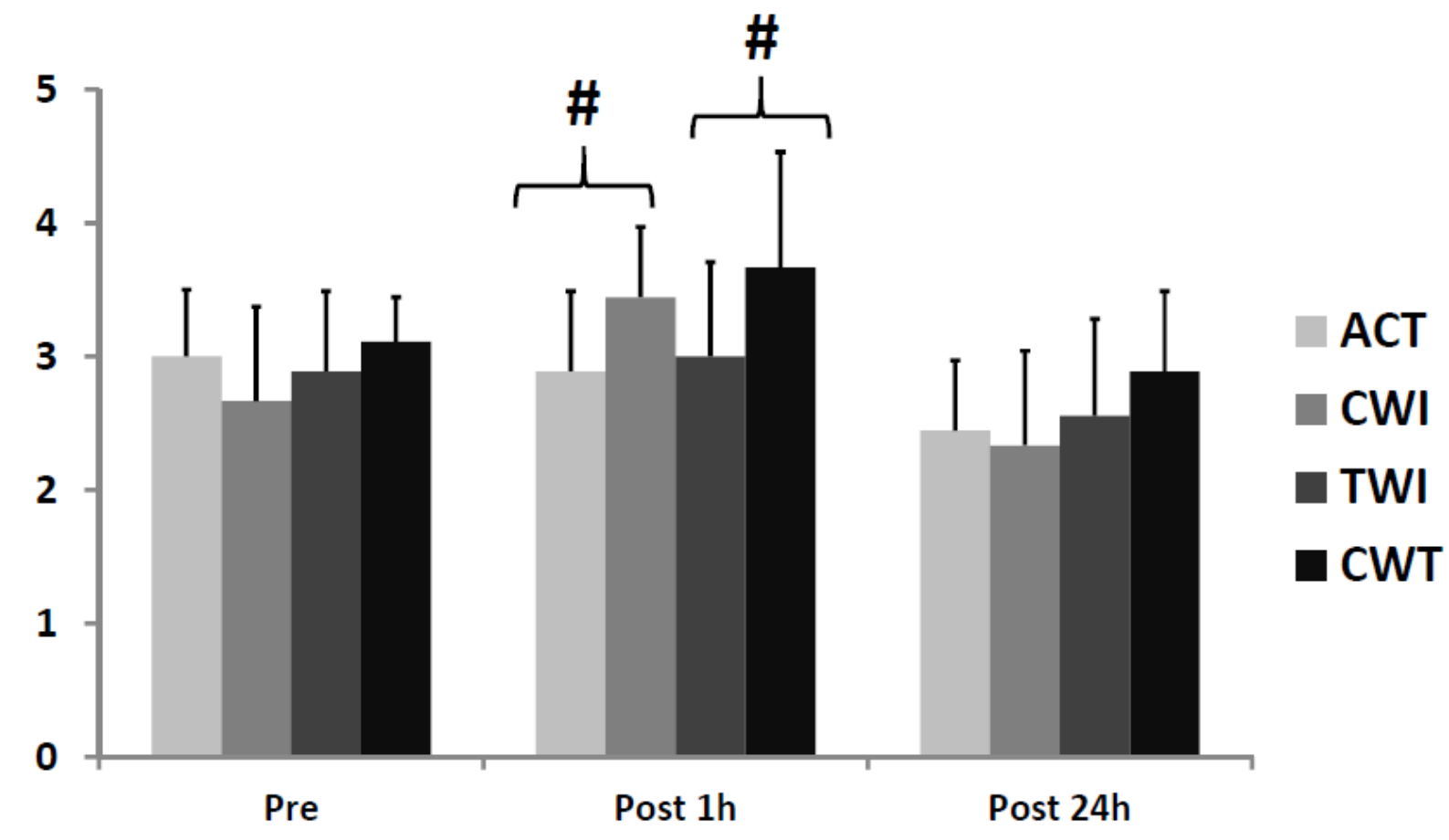

